Proceedings of the $2^{\text {nd }}$ ICEENG Conference, 23-25 Nov. 1999

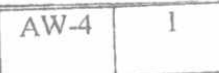

\author{
Military Technical College \\ Kobry Elkobbah, \\ Cairo, Egypt
}

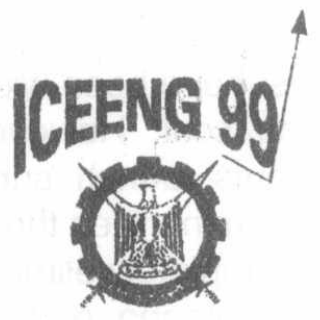
$2^{\text {nd }}$ International Conference on Electrical Engineering ICEENG 99

\title{
STUDY OF ENHANCEMENT METHODS FOR MICROSTRIP PATCH ANTENNA RADIATION PATTERNS USING FDTD METHOD
}

\author{
Ahmed N. Mahmoud', Maher M. Abd Elrazzak', IEEE Member, and \\ Hamdi A. Elmikati*, IEEE Senior Member
}

\begin{abstract}
In this paper, two techniques are presented for enhancement of the radiation patterns of a line-fed rectangular patch antenna. The two techniques are based on removal of the substrate under the patch or etching spaced periodic dielectric or air holes in the substrate. The finite-difference time-domain (FDTD) method with perfect matched layer absorber (PML) is applied to determine the return loss, the effective dielectric constant, the input impedance, and the radiation pattern of the proposed structure. The results obtained are found to be in good agreement with the published measured data.
\end{abstract}

\section{KEY WORD}

FDTD,PML, and Patch antenna

\footnotetext{
- Facluty of Eng., Mansoura Univ., El-Mansoura, Egypt. email: maher@ieee.org
} 


\section{INTRODUCTION}

In recent years, microstrip antennas have been widely used due to their simplicity, conformability and low manufacturing cost. However, a common feature of these antennas is that the antenna elements launch surface wave modes[1]. Although reducing the thickness of the substrate minimizes the amount of power launched into the surface waves[2], it does not completely eliminate them. Attempts to minimize the effect of surface waves on the antenna pattern are described in [3]. These consisted of trying to prevent radiation from the substrate edges by covering them with absorber or tape. Elimination of the surface waves would not only enhance the radiation pattern, but also increase the antenna efficiency.

In this work, two basic approaches are presented to overcome the above limitations. The first approach consists of removing most of the substrate beneath the patch, while the second approach consists of etching or drilling periodic dielectric or air holes in the substrate [4]. The finite-difference time-domain (FDTD) method is applied to the analysis of the patch antenna under consideration. This technique provides a lot of attractive advantages: easy programming, obvious stability condition, and minimum computational complexity[5-7]. The technique was initially formulated by Yee[5]. The basic idea of this technique consists of a direct discretization of the differential form of Maxwell's equations in time and space domains using second order accurate centeral differences. The resulting difference equations are then solved in a time marching sequence by alternately calculating the electric and magnetic fields on an interlaced Cartezian grid. The method was applied successfully for a variety of antenna configurations[8-9]. The solution of radiation problems require the use of absorbing boundary conditions (ABCs) to accurattely terminate the computational domain, and reduce reflections within the region of interest. The perfectly matched layer absorbing boundary conditions (PML $A B C$ ) are used to used to truncate FDTD computational domain [10].

\section{FORMULATION OF THE PROBLEM}

\section{II.A Basic FDTD principles}

This section presents the basic formulation of FDTD method. The geometry of the microstrip structure is shown in Fig. 1, where the strips and the bottom plane are perfect conductors with zero thickness. The substrate has a dielectric constant $\varepsilon_{\mathrm{r}}$.

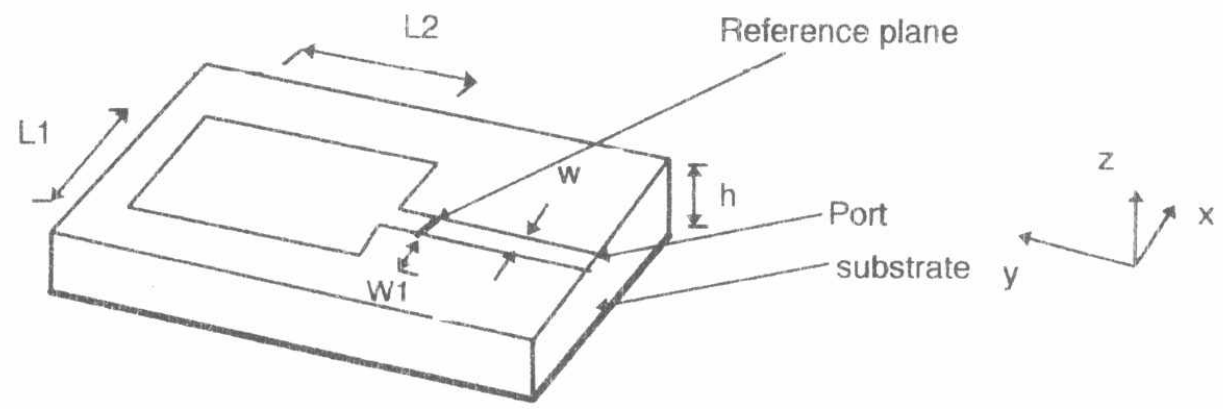

Fig. 1. Line-fed rectangular patch antenna. 
For this structure, Maxwell's equations can be written as [ 5],

$$
\begin{aligned}
& \frac{\partial \overrightarrow{\mathrm{H}}}{\partial \mathrm{t}}=-\frac{1}{\mu} \nabla \mathrm{x} \overrightarrow{\mathrm{E}}-\frac{\rho^{\prime}}{\mu} \overrightarrow{\mathrm{H}} \\
& \frac{\partial \overrightarrow{\mathrm{E}}}{\partial \mathrm{t}}=\frac{1}{\varepsilon} \nabla \mathrm{x} \overrightarrow{\mathrm{H}}-\frac{\sigma}{\varepsilon} \overrightarrow{\mathrm{E}}
\end{aligned}
$$

where,

$\rho^{\prime}$ is the magnetic resisitivity of the medium in $(\Omega / \mathrm{m})$

$\sigma$ is the electric conductivity of the medium in $(\mathrm{S} / \mathrm{m})$

$\frac{\partial}{\partial t}[\bullet]$ is the partial derivative with respect to time

Equations (1) are approximated using second order central difference scheme in space and time [5-7]. The position of E-and H-nodes are off in space by $\Delta / 2$ space, applying conventional FDTD method, the difference equations for the six field components in Cartesian coordinate of equation (1) are given, for instance, in references[6] and [7]. For example, the discretized equations for $H_{x}$ and $E_{x}$ field components are,

$$
\begin{aligned}
& H_{x \mid i, j, k}^{n+1 / 2}=\left.\left.D_{a}\right|_{i, j, k} \cdot H_{x}\right|_{j, j, k} ^{n-1 / 2}+\left.D_{b}\right|_{i, j, k}\left\{\frac{\left.E_{y}\right|_{i, j, k+1 / 2} ^{n}-\left.E_{y}\right|_{i, j, k-1 / 2} ^{n}}{\Delta z}-\frac{\left.E_{z}\right|_{i, j+1 / 2, k} ^{n}-\left.E_{z}\right|_{i, j-1 / 2, k} ^{n}}{\Delta y}\right\} \\
& \left.E_{x}\right|_{i, j, k} ^{n+1}=\left.\left.C_{a}\right|_{i, j, k} \cdot E_{x}\right|_{i, j, j, k} ^{n}+\left.C_{b}\right|_{i, j, k} ^{n}\left\{\frac{\left.H_{z}\right|_{i, j+1 / 2, k} ^{n+1 / 2}-\left.H_{z}\right|_{i, j-1 / 2, k} ^{n+1 / 2}}{\Delta y}-\frac{\left.H_{y}\right|_{i, j, k+1 / 2} ^{n+1 / 2}-\left.H_{y}\right|_{i, j, k-1 / 2} ^{n+1 / 2}}{\Delta z}\right\}
\end{aligned}
$$

where $\Delta x, \Delta y$, and $\Delta z$ are the space steps in the $x, y$, and $z$ directions respectively, the D's, and the C's are the updating electric and magnetic field components coefficients, respectively [6].

To ensure that the numerical error generated in one step does not accumulate and grow, a stability condition is usually applied. According to Courant, this condition is given by [6-7],

$$
\Delta \mathrm{t} \leq \frac{1}{\mathrm{v}_{\max }}\left(\frac{1}{\Delta \mathrm{x}^{2}}+\frac{1}{\Delta \mathrm{y}^{2}}+\frac{1}{\Delta \mathrm{z}^{2}}\right)^{-\frac{1}{2}}
$$

where

$\Delta t \quad$ is the maximum time step that may be used,

$v_{\max }$ is the phase velocity in the computational domain

II.B Perfectly matched layer (PML) ABC

In this analysis the perfectly matched layer absorbing boundary conditions are used 
to terminate the computational domain. The basic idea of this technique is based on surrounding the computational domain of an open structure with an artificial lossy medium whose impedance is matched to free space for all frequencies and all incident angles. The conventional PML technique requires that the FDTD field components be split into two orthogonal components. Details of this algorithn may be found in references [6] and [10].

\section{ANALYSIS OF A LINE-FED RECTANGULAR PATCH ANTENNA III.A Frequency dependent characteristics}

In this section, the return loss, the effective dielectric constant and the input impedance of the patch antenna shown in Fig. 1 are obtained. The dimensions of this structure are the same as those used in reference [11], where the total mesh dimensions are $60 \times 100 \times 16$ in the $x, y$, and $z$ directions, respectively. The rectangular patch antenna has $L 1=32 \Delta x$, and $L 2:=40 \Delta y$. The length of the microstrip line from the source plane to the edge of the antenna is $50 \Delta \mathrm{y}$. The reference plane is $10 \Delta y$ from the edge of the patch. The width of microstrip line w is $6 \Delta x$, the displacement w1 is $5 \Delta x$, the substrate thickness $h$ is $3 \Delta z$, and $13 \Delta z$ are used to model the free space above the substrate. The spatial steps used for this structure are $\Delta x=0.389 \mathrm{~mm}, \Delta y=0.400 \mathrm{~mm}$, and $\Delta z=0.265 \mathrm{~mm}$. The substrate dielectric constant is 2.2 .

In the present analysis, a Gaussian pulse with unit amplitude is excited on the microstrip line at the edge of the computation domain i.e. at port 1 (source plane) as shown in Fig. 1. This pulse is given by:

where,

$$
\mathrm{E}_{z}(t)=e^{-\frac{\left(t-t_{o}\right)^{2}}{T^{2}}}
$$

$$
\begin{aligned}
& \Delta t=0.441 p s, \\
& T: \text { Gaussian half width }=15 p s, \\
& t_{0}=3 T
\end{aligned}
$$

Initially all the fields on the whole computation domain are set to zero. After the pulse has propagated away port 1, absorbing boundary conditions are implemented several cells from the source. The transient response are recorded at the reference plane until all the fields in the computation domain decay to a negligible steady-state value. Then the Fourier transformation is used to obtain the return loss, the effective dielectric constant and the input impedance of the patch antenna according to [11]. All the analysis are carried out using FDTD method with perfect matched layer absorber. The thickness of the PML absorber is taken as 8 cells.

To determine the return loss of the patch antenna, one must calculate the refelected wave at the reference plane. The numerical FDTD simulations is carried out iwice. First simulation is in the absence reference incident waveform propagating alorig the feeder. A second simulation is repeated in the presence of the patch and the total incident and reflected voltages at the reference plane is obtained. Then the reflected wave is computed as the difference between the second and the first results. Using 
the incident and reflected time domain waveforms the return loss can be evaluated [11].

The effect of using air dielectric under the patch either by etching air holes or removal all the substrate under it on the return loss, the effective dielectric constant and the input impedance of the antenna are studied. The computed return loss of the patch for the above cases are shown in Fig. 2. As shown in the figure, the FDTD results for the original patch are found to be in good agreement with the published and measured data in [11]. It is also observed that the resonant frequencies increase as the air dielectric areas under the patch increase and the return loss remains approximately the same.

Fig. 3 shows the effective dielectric constant for the above cases. It can be seen from the figure that effective dielectric constant begins to drop near the resonant frequencies. This could be due to the appearance of higher order modes. Also one can see that the effective dielectric constants converge to the theoretical (average) values of 1.6 as the frequency is increased greatly.

The real and imaginary parts of the input impedance of this antenna for the above cases are shown in Fig. 4 and Fig. 5 respectively. From these figures, it can be shown that at resonant frequencies the input impedances are pure real. Also one can see that the first resonant frequency exhibits large values of input impedance while the second resonant frequency exhibits small values of input impedance which is suitable to match the feeder of 50 ohms to the antenna [9].

The effect of using different dielectric holes of dielectric constants $\varepsilon_{\mathrm{r}}$ of $2,1.5$ and 1 on the return loss, the effective dielectric constant and the input impedance of the antenna are now investigated. These holes are distributed uniformly in the substrate. The computed return loss of the patch for the above cases is shown in Fig. 6. As shown in the figure, the resonant frequencies increase as the dielectric constant of the holes decreases and the return loss remains approximately the same. Fig. 7 shows the effective dielectric constant for the above cases of the patch. The real and imaginary parts of the input impedance of this antenna for the above cases are shown in Fig. 8 and Fig. 9 respectively. From these figures, it can be shown that at resonant frequencies the input impedances are pure real.

Next, the effect of the number of air holes etched on all the substrate of the antenna on the return loss, the effective dielectric constant and the input impedance of the antenna are studied. The computed return loss of the patch is shown in Fig. 10. This figure shows that as the air dielectric areas increase both the resonant frequencies and the return loss increase. Fig. 11 shows the effective dielectric constant which converges to the expected theoretical (average) values of 1.6 as the frequency is increased greatly. The real and imaginary parts of the input impedance of this antenna for the present case are shown in Fig. 12 and Fig. 13, respectively. From these figures, it can be shown that at resonant frequencies the input impedances are pure real and their values decrease as the number of air holes increase. 


\section{B Radiation patterns}

The goal of this section is to determine the radiation patterns of the antenna under consideration. The far-fields of the antenna can be obtained concurrently with the FDTD simulation through the powerful near -to far-field zone transformation principle given in $[6,7,12]$. The core of this process starts with the surface equivalence theorem, from which the equivalent electric and magnetic currents can be obtained on the virtual surface that completely encloses the antenna based on the near-field data. Once these equivalent currents are computed, one can calculate the radiation patterns using the radiation equations shown in Balanis's book [1,eqs. (12.10)-(1212)]. In the present work, a Gaussian pulse modelated by sinusoidal wave excitation near resonant frequency is used. As soon as the steady state conditions are reached, the normalized $\mathrm{E}$-plane and $\mathrm{H}$-plane field patterns at each far zone angle can be obtained.

Fig. 14 shows the calculated normalized E-plane and $\mathrm{H}$-plane field patterns for the antenna shown in Fig. 1 at the resonant frequency of $8 \mathrm{GHz}$. As shown in the figure, one can find good agreement between the FDTD results and the calculated data given by the approximate technique; the cavity model [1], especially in the $\mathrm{H}$-plane.

At first, the effect of using air dielectric under the patch either by etching air holes or removal all the substrate under it on the radiation pattern of the antenna is studied. Fig. 15 shows the E-plane and $\mathrm{H}$-plane field patterns for the antenna shown in Fig. 1 for the above cases at resonant frequencies. As shown in the figure, one can read that the E-plane pattern is improved as the areas of air dielectric under the patch increase since much of the radiation power is focused in the z-direction, also the beam width decreases. The narrower the beam width, the better action of focussing the power. For the $\mathrm{H}$-plane pattern of that antenna for the above cases one can see that there is no great difference for the different cases. Also one can see good similarities between both the properties of the E-plane and $\mathrm{H}$-plane field patterns mentioned above and those given in Fig. 1 in [4].

Secondly, the effect of using dielectric holes of different dielectric constants $\varepsilon_{\mathrm{r}}$ of 2 , 1.5 and 1 etched on all the substrate of the antenna on the radiation pattern is studied. Fig. 16 shows the E-plane and $\mathrm{H}$-plane field patterns for the antenna shown in Fig. 1 for these cases at resonant frequencies. From the figure, one can see that the E-plane pattern is improved as dielectric constant of the dielectric hole is decreased, while the $\mathrm{H}$-plane pattern remains approximately the same.

At last, the effect of using different numbers of air holes through all the substrate of the antenna is studied. The E-plane and $\mathrm{H}$-plane field patterns for the antenna shown in Fig. 1 for the above cases at resonant frequencies is shown in Fig. 17. From the figure, one can read that as the areas of dielectric air increase the E-plane field pattern is improved while the $\mathrm{H}$-plane pattern remains approximately the same.

\section{CONCLUSION}

In this paper, we have presented two different techniques to improve the radiaticn 
patterns of a line- fed rectangular patch antenna. These techniques are based on removal of the substrate under the patch or etching spaced periodic dielectric or air holes in the substrate. The removing all of the substrate under the patch increases the efficiency and directivity since much of the radiation power is focused in a desired direction. The finite- difference time- domain FDTD method with perfect matched layer has been used to determine the return loss, the effective dielectric constant, the input impedance and the radiation pattern of the patch. This technique provides a lot of attractive advantages: easy programming, obvious stability condition, and minimum computational complexity. The results obtained by the FDTD with perfectly matched layer absorber are found to be in good agreement with the published measured data.

\section{REFERENCES}

[1] C. A. Balanis, Antenna Theory: Analysis and Design, New York, John Wiley \& Sons Inc. (1997).

[2] N. G. Alexopoulos, P. B. Katethi, and D.B. Rutledge," Substrate optimization for integrated circuit antennas," IEEE Trans., Microwave Theory Tech. vol. MTT-31, No.7, pp. 550-557, (1983).

[3] D. H. Schaubert and K. S. Yngvessen, "Experimental study of the microstrip array on high permittivity substrate," IEEE Trans. Antennas Propagat.,vol.34. No.1, pp.92-97,(1986).

[4] M. J. Vaughan, K.Y. Hur, and R. C. Compton,"Improvement of microstrip patch antenna radiation patterns," IEEE Trans. Antennas Propagat.,vol.42, No.6, pp.882 -885, (1994).

[5] K. S. Yee, "Numerical solution of initial boundary value problems involving Maxwell's equations in isotropic media," IEEE Trans. Antennas Propagat.," vol. 14, pp.302-307, (1966).

[6] Allen Taflove, Computational Electrodynamics: The Finite-Difference TimeDomain Method, Artech House, Boston, London.(1995).

[7] Karl. S. Kuenz and Raymound J. Luebbers, The Finite- Difference Time-Domain Method for Electromagnetics, CRC press, London- Tokyo, (1993).

[8] A. D. Olver, and U. Sterr, "Design of corner reflector antennas using FDTD," IEE Proc. Microw, Antenna Propagat., vol. 145, No. 6, pp.481-485, (1998).

[9] K. D. Katsiba, C. A. Balanis, P. A.A. Tirkas, and C. r. Birctcher," Folded loop antenna for mobile hand-held units," IEEE Trans. Antennas Propagat.,vol.46, No.2, pp.260 -266, (1998).

[10] Jean - Pierre Berenger," A perfectly matched layers for the FDTD solution of wave- structure interaction problems," IEEE. Trans. Antennas Propagat.,vol.44, No. 1, pp.110-117, (1996).

[11] D. M. Sheen, S. M. Ali, M. D. Abouzahra, and J. A.Kong, "Application of the three-dimensional finite-difference time-domain method to the analysis of planar microstrip circuits," IEEE Trans., Microwave Theory Tech. vol. MTT-38, No.7, pp. 849-857, (1990).

[12] T. Martin, "An improved near- to far- zone transformation for the finitedifference time -domain method," IEEE. Trans. Antennas Propagat.,vol.46, No. 9, pp.1263-1270, (1998). 


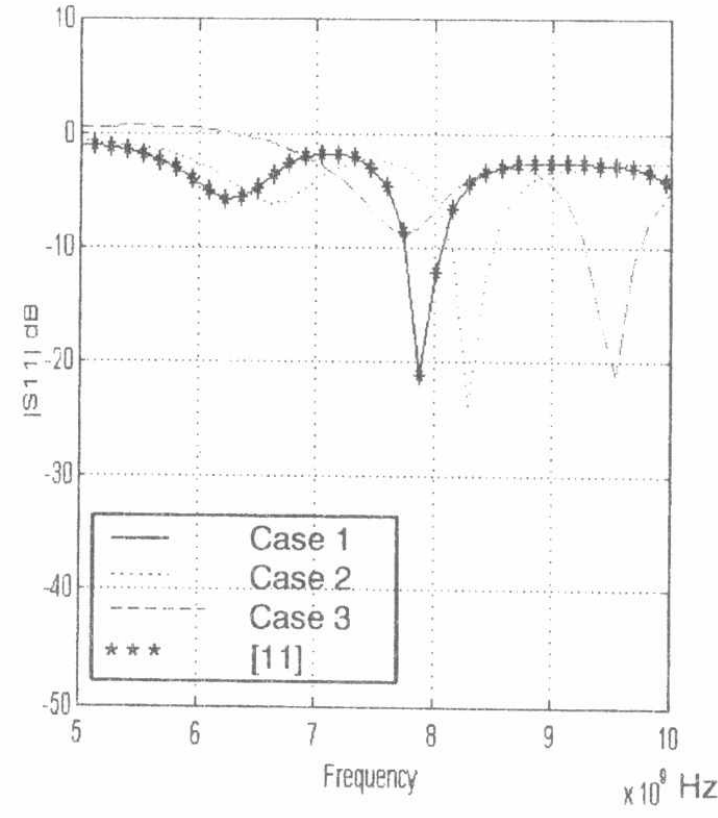

Fig. 2. The return loss of the patch antenna shown in Fig.1. Case 1: original patch; Case 2: 9air holes under patch; Case3: all air under patch.

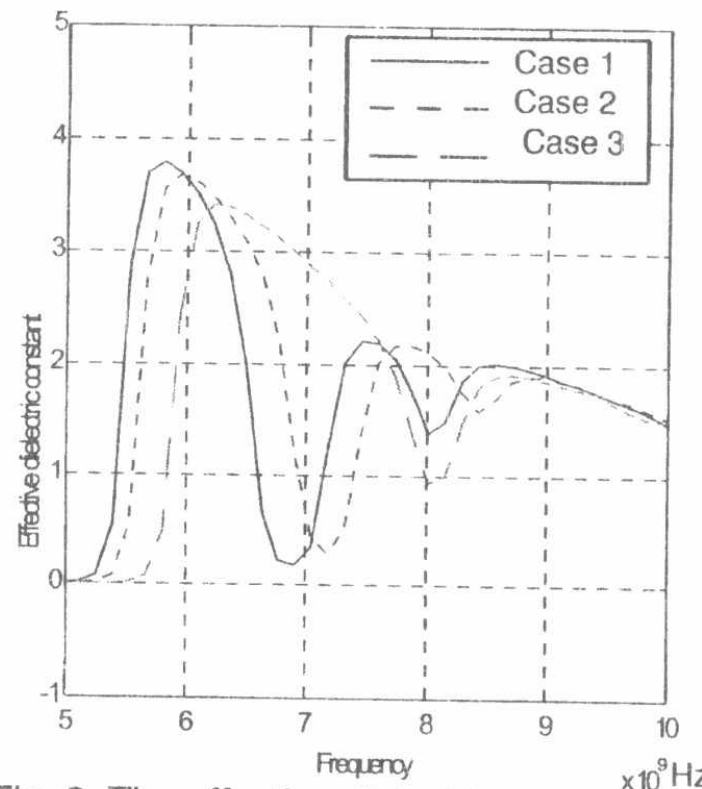

Fig. 3. The effective dielectric constant of the patch shown in Fig.1. Case 1: original patch; Case 2: 9air holes under patch; Case3: all air under patch.

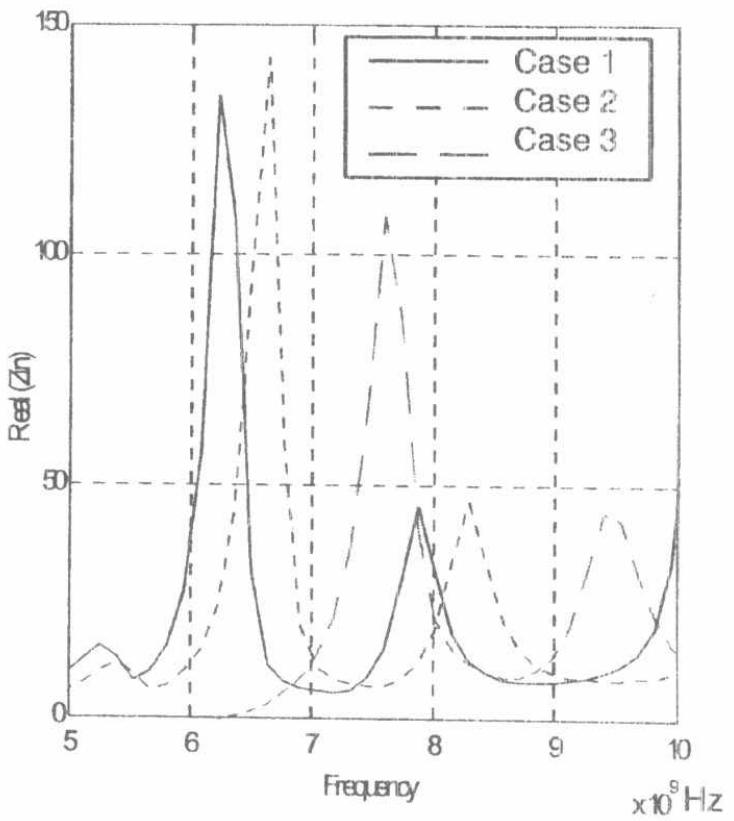

Fig. 4. The real part of the input impedance of the patch shown in Fig. 1. Case 1: original patch; Case 2: 9air holes under patch; Case3: all air under patch.

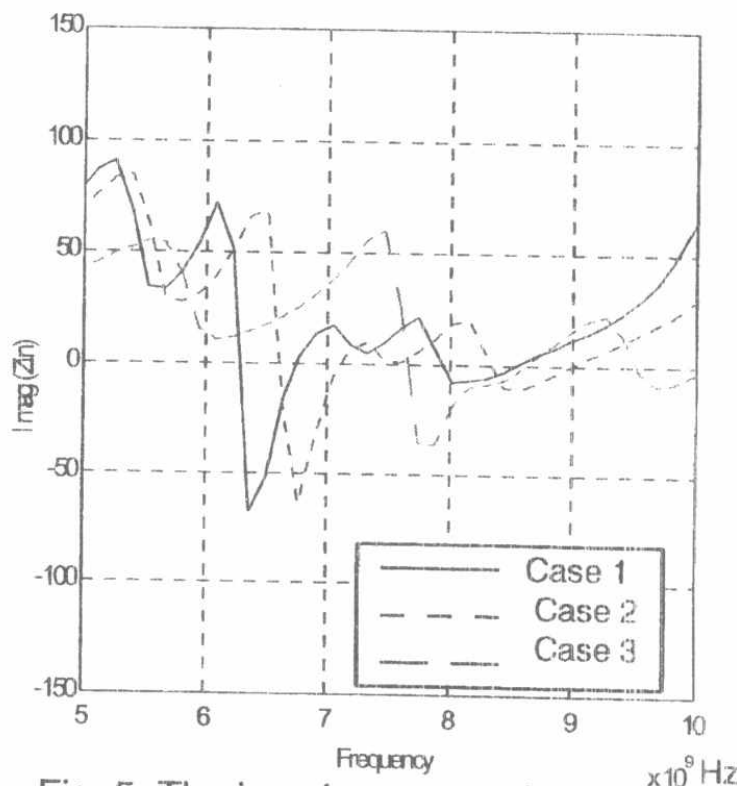

Fig. 5. The imaginary part of the input impedance of the patch shown in Fig.1. Case 1: original patch; Case 2: 9air holes under patch; Case3: all air under patch. 


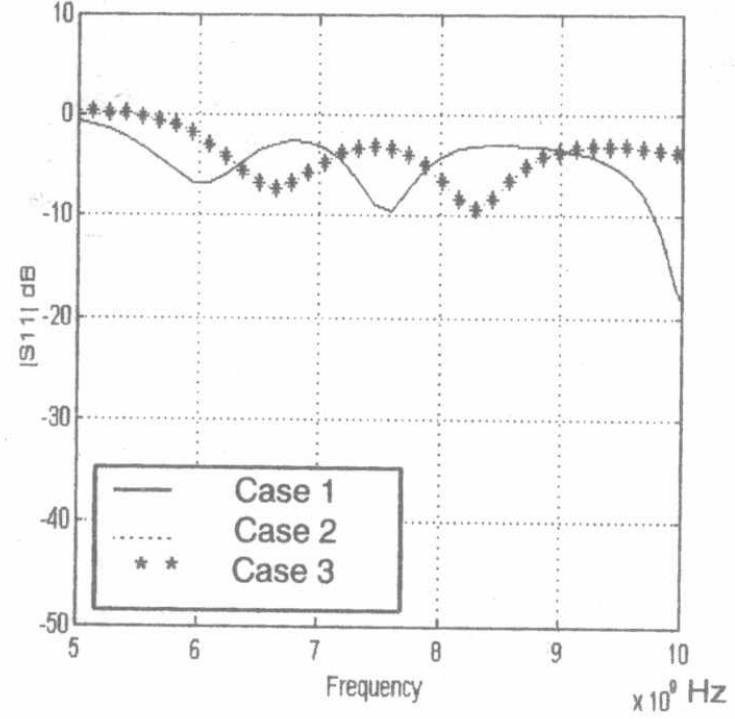

Fig. 6. The return loss of the patch antenna shown in Fig. 1 for 50 holes etched on all the substrate. Case 1: dielectric holes of epr=2; Case 2: dielectric holes of epr=1.5; Case 3: air holes.

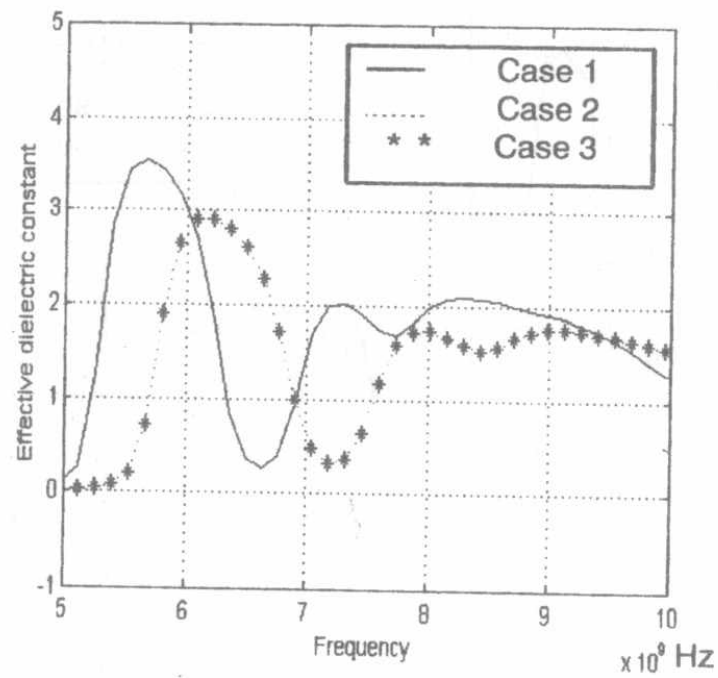

Fig. 7. The effective dielectric constant of the patch shown in Fig. 1 for 50 holes etched on all the substrate. Case 1: dielectric holes of epr=2; Case 2: dielectric holes of epr=1.5; Case 3: air holes.

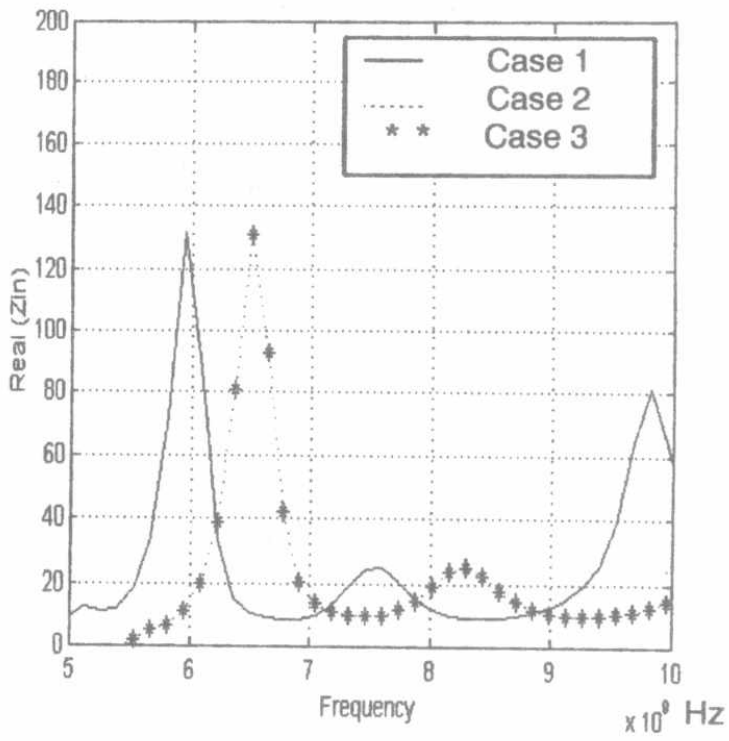

Fig. 8. The real part of the input impedance of the patch shown in Fig. 1 for 50 holes etched on all the substrate. Case 1: dielectric holes of epr=2; Case 2: dielectric holes of epr=1.5; Case 3: air holes.

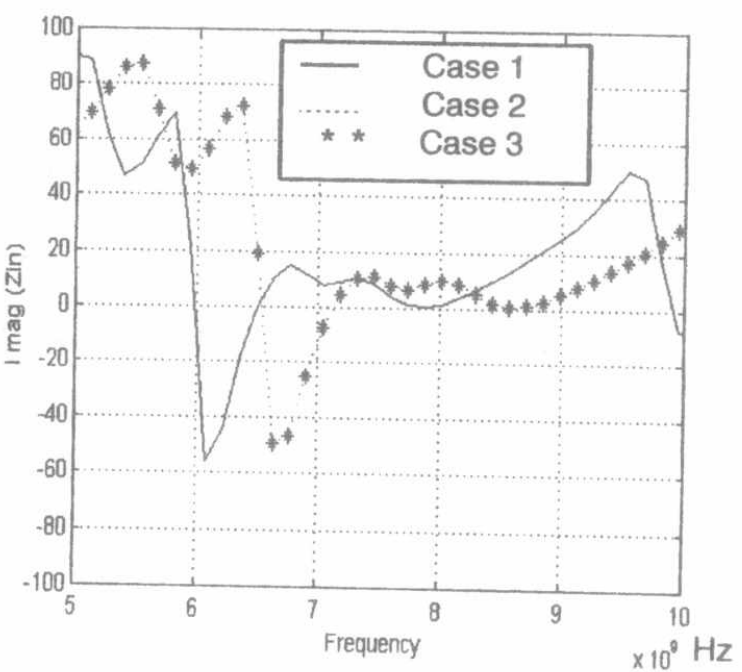

Fig. 9. The imaginary part of the input impedance of the patch shown in Fig.1 for 50 holes etched on all the substrate. Case 1: dielectric holes of epr=2; Case 2: dielectric holes of epr=1.5; Case 3: air holes. 


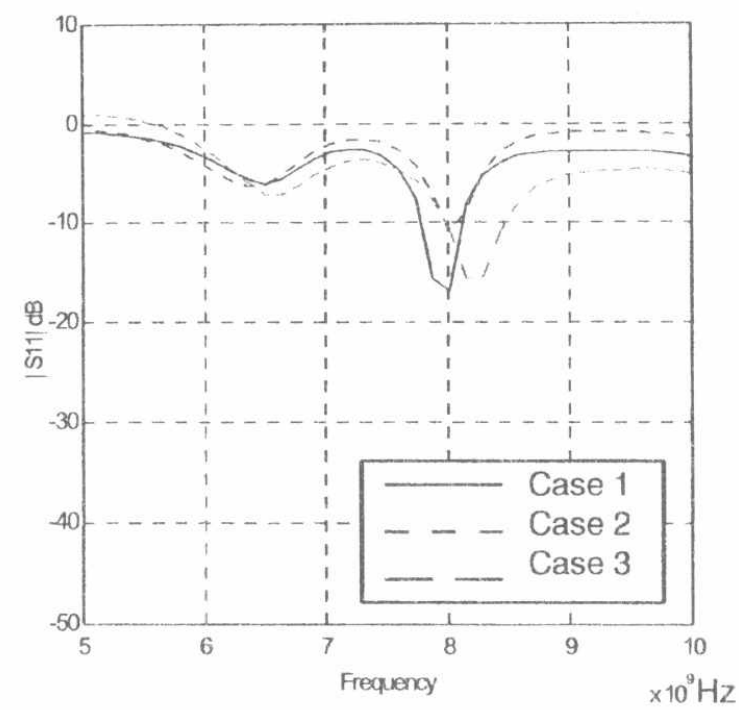

Fig. 10. The return loss of the patch shown in Fig. 1 for different air holes etched on all the substrate. Case 1: 9 holes; Case 2: 25 holes; Case 3: 35 holes.

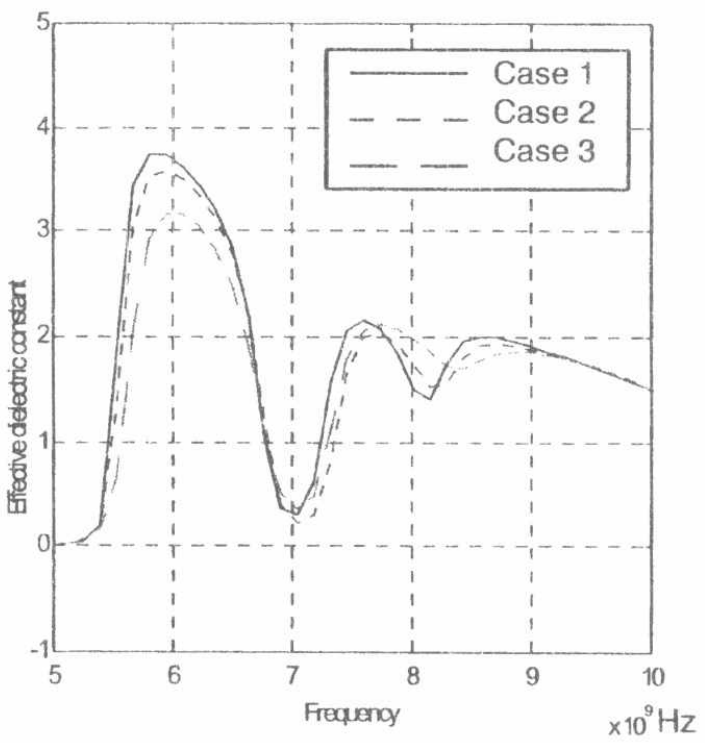

Fig. 11. The effective dielectric constant of the patch shown in Fig. 1 for different air holes etched on all the substrate. Case 1: 9 holes; Case 2: 25 holes; Case 3: 35 holes.

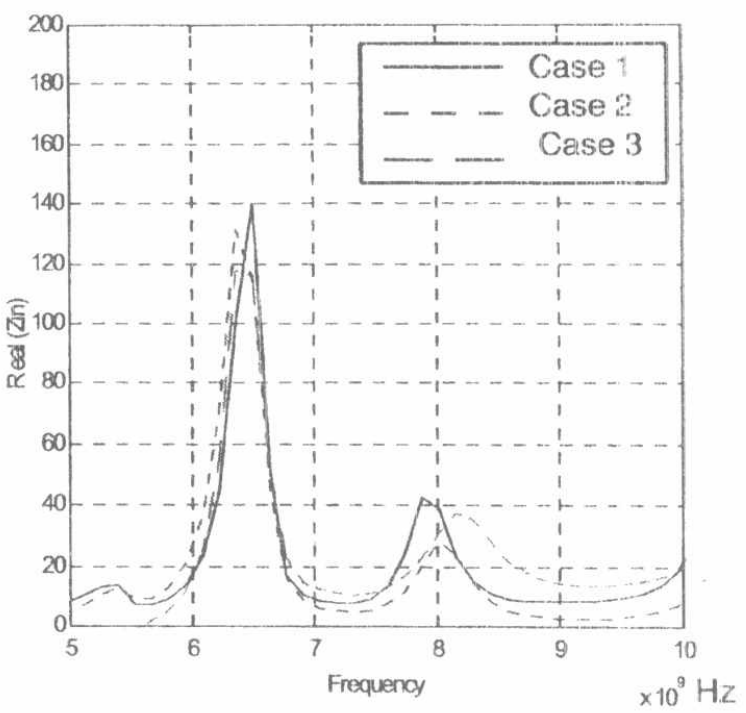

Fig. 12. The real part of the input impedance of the patch shown in Fig. 1 for different air holes etched on all the substrate. Case 1: 9 holes; Case 2:: 25 holes; Case 3: 35 holes.

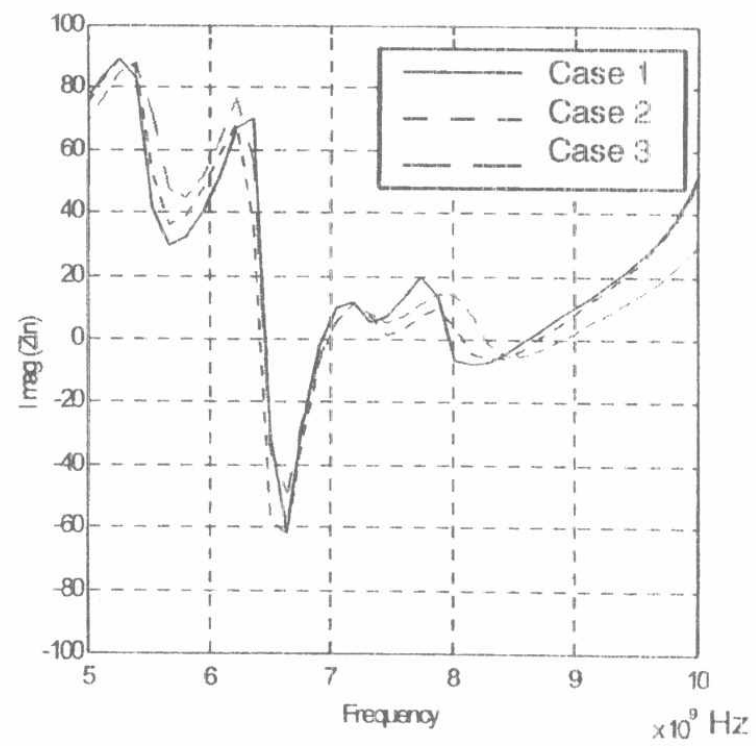

Fig. 13. The imaginary part of the input impedance of the patch shown in Fig. 1 for different air holes etched on all the substrate. Case 1: 9 holes; Case 2: 25 holes; Case 3: 35 holes. 


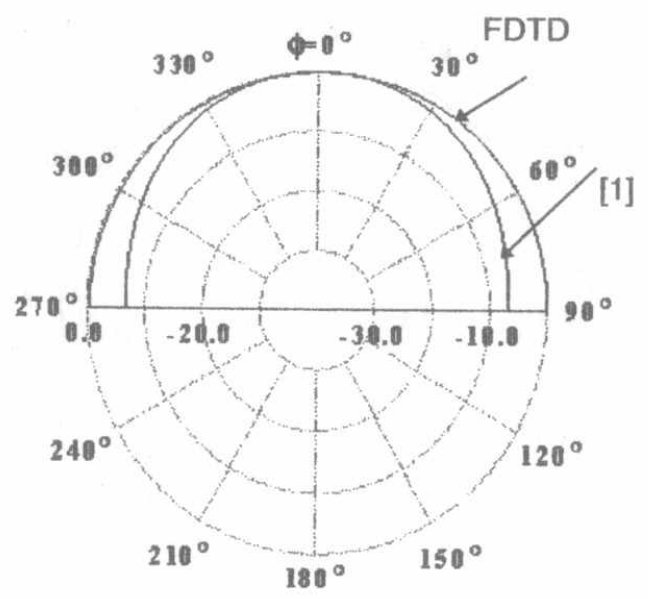

(a)

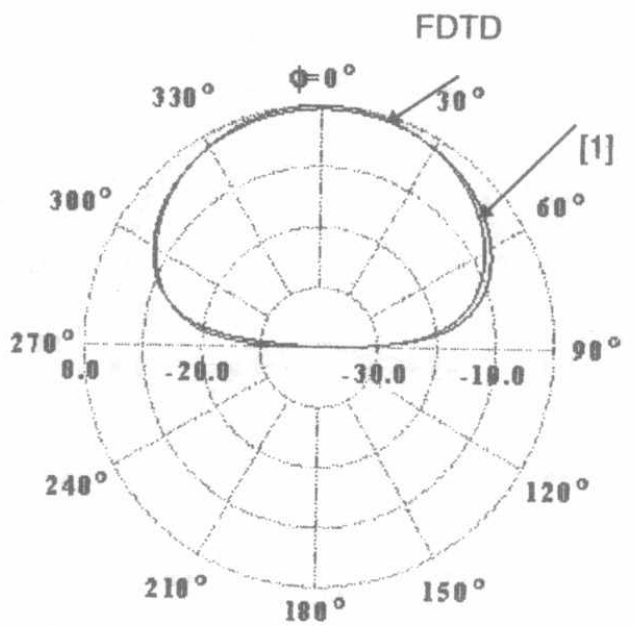

(b)

Fig. 14. Radiation field patterns for the patch shown in Fig. 1at $f_{0}=8 G H z$. (a) $E$ plane; (b) H-plane.

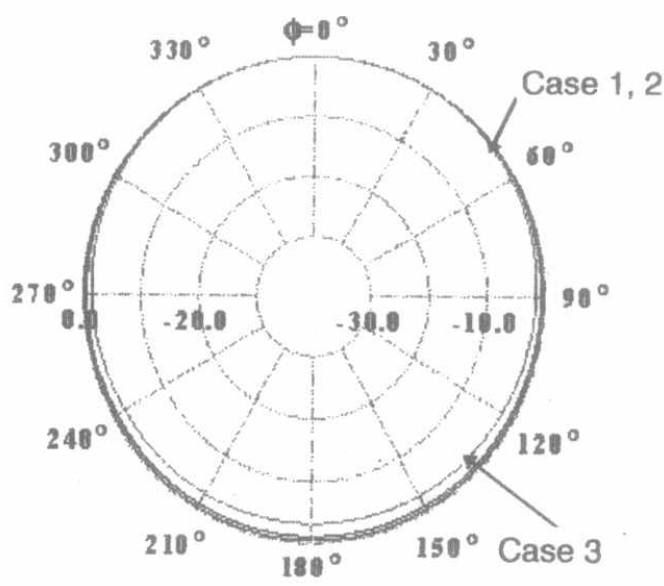

(a)

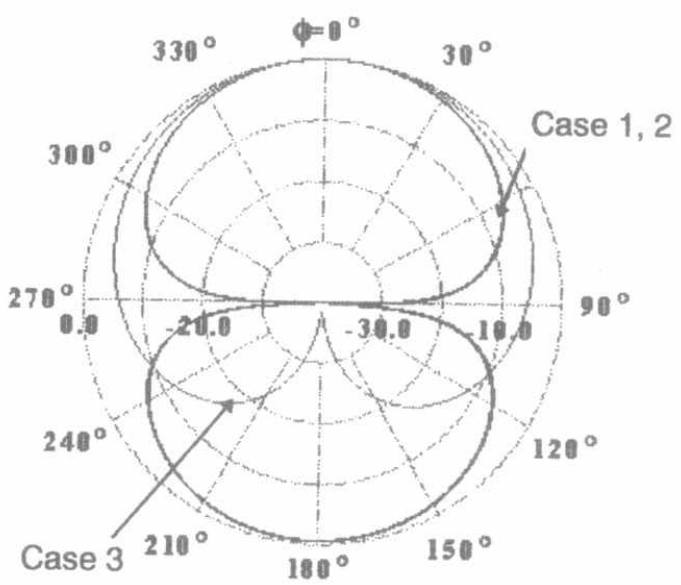

(b)

Fig. 15. Radiation field pattern of the patch shown in Fig. 1. Case 1: normal patch; Case 2 : 9air holes under patch; Case 3: all air under patch. (a) E-plane;

(b) H-plane. 


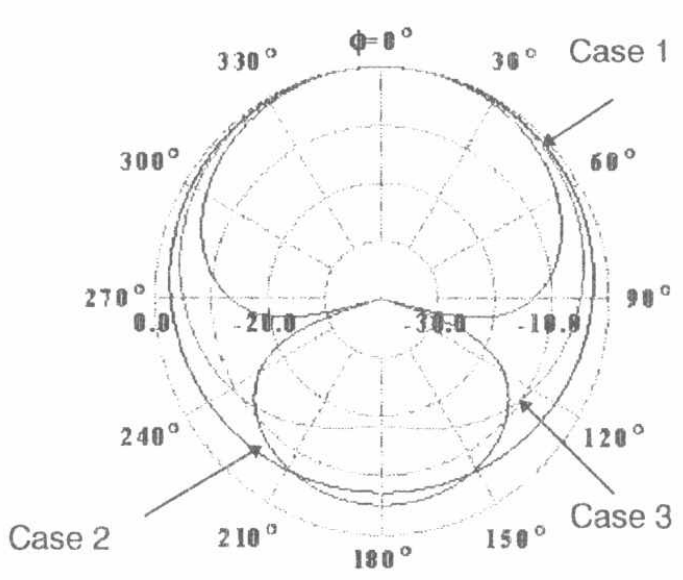

(a)

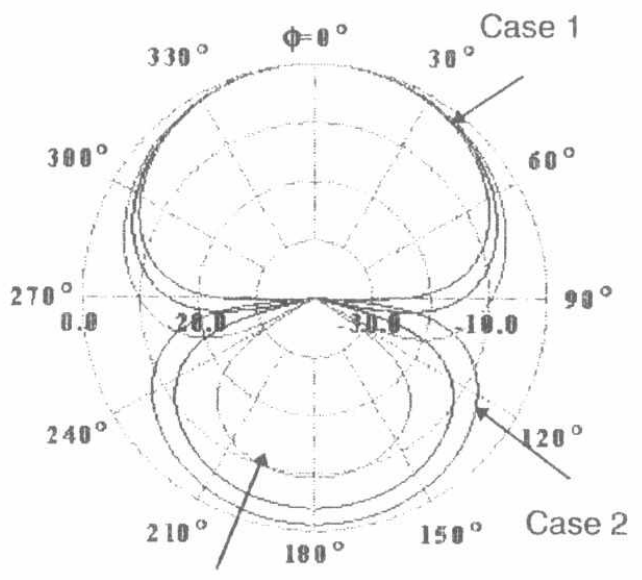

Case 3

(b)

Fig. 16. Radiation field pattern of the patch shown in Fig. 1 for 50 holes etched on all the substrate Case 1: dielectric holes of epr=2; Case 2: dielectric holes of epr=1.5; Case 3: air holes. (a)E-plane; (b) $\mathrm{H}$-plane.

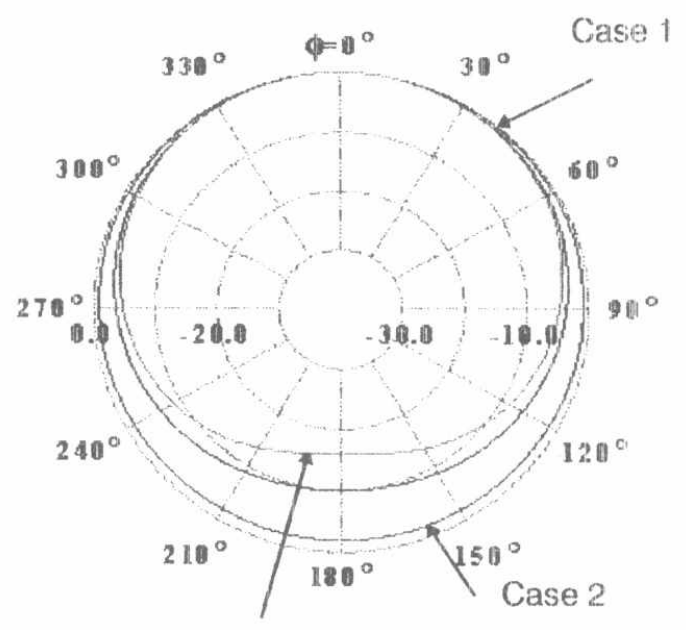

Case 3

(a)

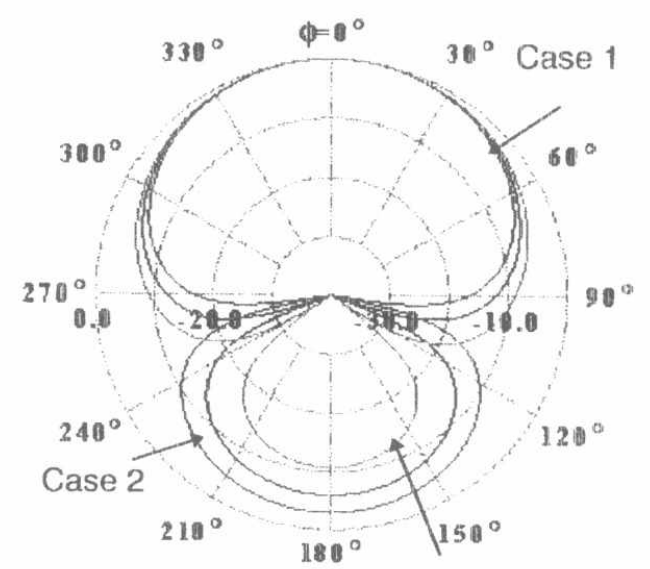

(b)

Fig. 17. Radiation field pattern of the patch shown in Fig. 1 for different air holes etched on all the substrate. Case 1: 9 holes; Case 2: 25 holes; and Case 3: 35 holes. (a)E-plane; (b)H-plane. 